\title{
Enhancing Success in STEM: Spatial Visualization and Freehand Sketching with the SpatialKidsTM iPad App
}

\section{Dr. Nathan Delson, University of California, San Diego}

Nathan Delson, Ph.D. is a Teaching Professor at the University of California at San Diego. His interests include robotics, biomedical devices, product design, and engineering education. He is co-founder of Coactive Drive Corporation, a company that provides force feedback solutions. Since 1999 he has taught engineering design and been the director of the Mechanical Engineering Design center at UC San Diego. In fall 2012, Dr. Delson introduced a Product Design \& Entrepreneurship course, where students develop their own product concepts.

Dr. Yael Van Den Einde, University of California, San Diego 


\section{WORKSHOP PROPOSAL FORM}

2015 Annual ASEE K-12 Workshop on Engineering Education

"Authentic Engineering: Representing \& Emphasizing the E in STEM"

Presented by Dassault Systems

Saturday, June 13, 2015

8:00 A.M. - 5:00 P.M.

Sheraton Seattle $\mid$ Seattle $\mid$ WA

Please complete this form, save it as a PDF file only and upload it through the ASEE Paper Management system as shown in the K12 Workshop Presenter's Kit.

All notifications will be by email from the ASEE Paper Management system.

NOTE: To ensure that emails are not obstructed by spam blockers, please make sure to WHITELIST the email addresses: monolith@asee.org and conferences@,asee.org and s.harrington-hurd@,asee.org.

Direct questions to Stephanie Harrington-Hurd, ASEE K-12 Activities Manager, at s.harringtonhurd@asee.org. Additional workshop details are available at: http://www.asee.org/K12Workshop. Thank you!

\section{Deadline}

Friday, January 23, 2015 by 5:00PM EST

Presenters will be notified of acceptance status by March 14. Late submissions will not be accepted. Advanced Workshop Registration will open December 6, 2013.

\section{SUBMISSION INFORMATION}

Provide the first and last name of each presenter, including affiliations. If there is more than one presenter, designate one person as the organizer and provide only that person's contact information. The organizer is responsible for communicating to co-presenters.

Number of Presenters: 2

Presenter Name(s):

1) Last Delson First Nathan Affiliation Univ. of California, San Diego

2) Last Van Den Einde $\quad$ First Lelli Affiliation Univ. of California, San Diego

3) Last First Affiliation

Contact Person's Name: Nathan Delson

Contact Person's Email: ndelson@ucsd.edu

Contact Person's Phone: (858) 534-0655

Contact Person's Alternate Phone: (858) 395-7220 


\section{WORKSHOP PROPOSAL FORM}

2015 Annual ASEE K-12 Workshop on Engineering Education

"Authentic Engineering: Representing \& Emphasizing the E in STEM"

Presented by Dassault Systems

Saturday, June 13, 2015

8:00 A.M. - 5:00 P.M.

Sheraton Seattle $\mid$ Seattle $\mid$ WA

Please provide a one-paragraph bio for each presenter (in the order listed above). The bio should not exceed 70 words and should be written as you would want it to appear on the ASEE website and program materials.

1) Nathan Delson is a Teaching Professor at UC San Diego. His interests include robotics, biomedical devices, product design, and engineering education. He is co-founder of Coactive Drive Corporation, a company that provides force feedback solutions. Since 1999 he has taught engineering design and been the director of the Mechanical Engineering Design center. In 2012, Dr. Delson introduced a Product Design \& Entrepreneurship course, where students develop their own product concepts.

2) Van Den Einde is a Teaching Professor in Structural Engineering at UC San Diego. She incorporates education innovations into courses (Peer Instruction, Project-based learning), prepares next generation faculty, advises student organizations, hears cases of academic misconduct, is responsible for ABET, and is committed to fostering a supportive environment for diverse students. Her research focuses on engagement strategies for large classrooms and developing K-16 curriculum in earthquake engineering and spatial visualization.

3)

\section{WORKSHOP INFORMATION}

\section{Proposed Title:}

Enhancing Success in STEM: Spatial Visualization and Freehand Sketching with the SpatialKids $^{\mathrm{TM}}$ iPad App

Abstract: Please provide a concise description that includes the workshop's learning objectives (maximum 750 characters). The abstract is used on the ASEE website, program materials, and otherK-12 Workshop promotional activities.

Spatial Visualization (SV) is the mental representation and manipulation of 2D and 3D shapes. Skills in SV and Freehand Sketching have been correlated to success in STEM, yet SV is not formally part of K-12 curriculum. An interactive SV drawing application (SpatialKids ${ }^{\mathrm{TM}}$ ) geared towards K-8 grades has been developed at UC San Diego using touchscreen interface technology on an iPad. In this interactive workshop, teachers will learn 1) SV skills such as 2D rotations, 3D isometric views, and 2D orthographic projections; 2) how to use the iPad app including the algorithm that automatically grades students' sketches and provides guidance when requested, and 3 ) best practices for how to implement the SV training in their curriculum.

Workshop Description. Please provide a detailed description of the proposed workshop that, at minimum, explicitly addresses the following (maximum 4,000 characters): 


\section{WORKSHOP PROPOSAL FORM}

2015 Annual ASEE K-12 Workshop on Engineering Education

"Authentic Engineering: Representing \& Emphasizing the E in STEM"

Presented by Dassault Systems

Saturday, June 13, 2015

8:00 A.M. - 5:00 P.M.

Sheraton Seattle $\mid$ Seattle $\mid$ WA

a. Learning objectives

b. Hands-on activities and interactive exercises

c. Materials that participants can take with them

d. Practical application for teachers and outreach staff

Learning objectives: In this workshop, participants will use a touchscreen device (iPad) to engage in interactive spatial visualization (SV) activities using the SpatialKids ${ }^{\mathrm{TM}}$ App developed at UC San Diego. Learning objectives for the workshop are to teach participants SV skills such as $2 \mathrm{D}$ rotations, sketching of $3 \mathrm{D}$ isometrics and $2 \mathrm{D}$ orthographic projections, and flat pattern folding; how to use the iPad app including the feature that automatically grades students' sketches and provides guidance when requested; and to provide participants with best practices for how to implement the SV training in their own classrooms. The touchscreen technology facilitates an engaging and cost-effective SV training method that allows freehand sketching with natural hand motions not possible with a computer mouse.

Hands-on Activities \& Interactive Exercises: Workshop participants will work directly with iPads provided by the presenters. We will walk through the various SV lessons and make use of the integrated tutorials and feedback system. An example of a free response exercise related to the ability to manipulate and rotate a shape one quarter turn counterclockwise is shown in Figure 1a. When a sketch is submitted for grading and there is an error in the solution, students are given the option to Retry the sketch, obtain a Hint (showing a video tutorial of the solution steps), or take a Peek at the solution and then redo the sketch. Figure 1b shows a screenshot of the message received once an activity containing an error in the solution has been submitted for grading, and Figure 1c shows the type of instantaneous feedback provided when the "Peek" option is selected. In this case, green lines indicate correct portions of the sketch, red solid lines indicate areas where incorrect lines are drawn, and red-hashed lines represent areas where lines should have been drawn but were omitted. 


\section{WORKSHOP PROPOSAL FORM}

2015 Annual ASEE K-12 Workshop on Engineering Education "Authentic Engineering: Representing \& Emphasizing the E in STEM"

Presented by Dassault Systems

Saturday, June 13, 2015

8:00 A.M. - 5:00 P.M.

Sheraton Seattle $\mid$ Seattle $\mid$ WA

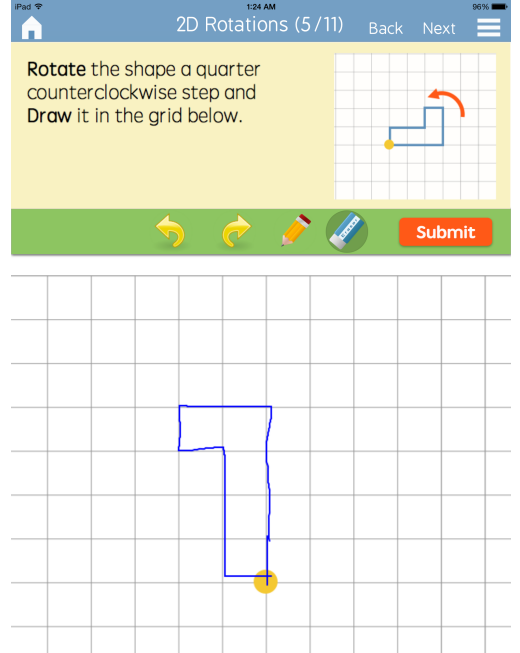

a) 2D rotation activity

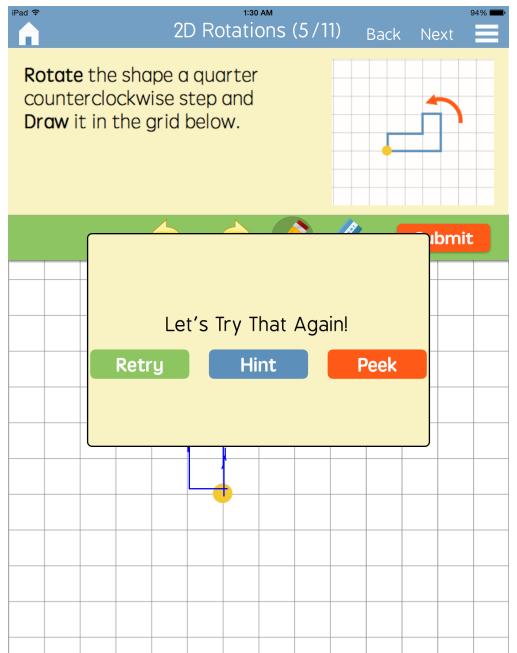

b) Grading algorithm response

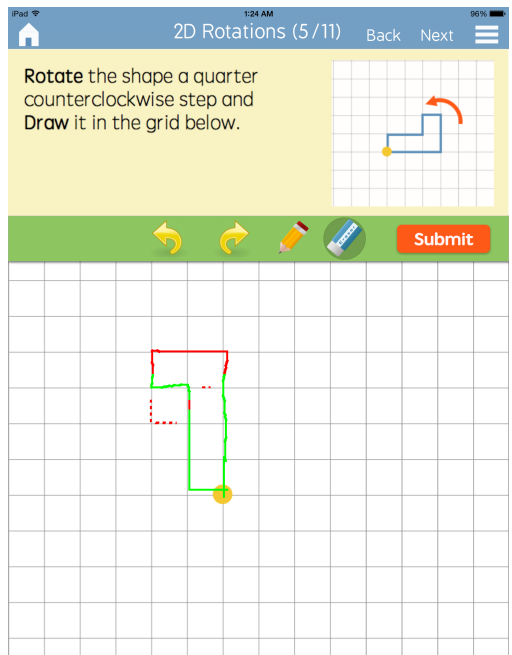

c) Feedback during Peek Figure 1: SpatialKids ${ }^{\text {TM }}$ screenshots

Each lesson in the software begins with easy problems, and gradually increments in difficulty. Thus, students are able to work independently and can use the Hint and Peek features to resolve most problems on their own. A point system encourages students to complete assignments without Hints or Peeks as they progress. At the end of each lesson a few test questions are provided where the Hint and Peek questions have been disabled. Teachers can view each student's progress including the number of assignments completed as well as how often the Hint and Peek features have been used.

Materials that participants can take with them: Workshop participants will be given a license to the iPad App to use on their own devices. For those that do not have a classroom set of iPads available at their home institutions, a web-based version is under development for use on Windows, Chrome, and Android devices. A license will be provided when it becomes available.

Practical application for teachers and outreach staff: SpatialKids ${ }^{\mathrm{TM}}$ is aligned with the engineering habits of mind, as well as K-8 common core and NGSS standards (see below). The app can be integrated directly into math, science or engineering curriculum, or can be introduced to students through outreach events. For example, the app was incorporated into a "Girls in Engineering" day sponsored by the Stanley E. Foster School of Engineering, Innovation, and Design in San Diego. Middle school girls were invited to explore the design process through an engineering and architecture design challenge. If they finished their designs early, they were given the opportunity to play with the SpatialKids ${ }^{\mathrm{TM}}$ App to test their SV skills and ultimately excite them about STEM. Feedback from this event was extremely positive.

Authentic Engineering Connection. Identify and describe how you will explicitly address the ways in which your lesson or activity is representative of the processes, habits of mind and practices used by engineers, or is demonstrative of work in specific engineering fields. ${ }^{i}$ At least 


\section{WORKSHOP PROPOSAL FORM}

2015 Annual ASEE K-12 Workshop on Engineering Education "Authentic Engineering: Representing \& Emphasizing the E in STEM"

Presented by Dassault Systems

Saturday, June 13, 2015

8:00 A.M. - 5:00 P.M.

Sheraton Seattle $\mid$ Seattle $\mid$ WA

one of those must be within the first four listed, below; i.e., do not only check "other". Check all that apply:

Use of an engineering design process that has at least one iteration/improvement

$\triangle$ Attention to specific engineering habits of mind

$\triangle$ Attention to engineering practices (as described in the NGSS/Framework and as practiced by engineers)

Attention to specific engineering careers or fields related to the lesson/activity

$\triangle$ Other (please describe below)

Provide a description of how you will explicitly address these aspects of authentic engineering in your workshop (maximum 2,000 characters):

Authentic Engineering: Drawing is a fundamental engineering skill that is used for concept generation and sharing of ideas with others. The proverbial "back of the envelope" sketch is something engineers do on a daily basis, and is also something that is highly engaging for young children with their love of drawing. This workshop introduces students to some of the technical aspects of engineering drawings and develops SV skills, which have been shown to help with performance in STEM.

Engineering Habits of Mind: The activities presented in this workshop are representative of Creativity, Optimism, and Communication. Sketching is an integral part of the brainstorming and creative process. Optimism is increased when students are able to visualize design solutions and draw how they will look. We all know that "a picture is worth 1000 words," and thus sketching is integral to technical communication.

Engineering practices based on NGSS Framework: Engineering, technology and the application of science is one of the discipline core ideas in NGSS. The SpatialKids ${ }^{\mathrm{TM}}$ App teaches important skills used in the engineering design process; it provides skills that enable students to brainstorm and sketch (in 3D) possible solutions to a problem, communicate with peers about proposed solutions, and share ideas that lead to improved designs (e.g., 3-5-ETS1-2).

Common Core: SpatialKids ${ }^{\mathrm{TM}}$ aligns with geometry standards. In particular, Kindergarten (analyze, compare, create, and compose shapes); $1^{\text {st }}$ grade (build, draw and reason with shapes and their attributes), $5^{\text {th }}$ grade (classify $2 \mathrm{D}$ figures based on properties), $6^{\text {th }}$ grade (represent $3 \mathrm{D}$ figures using nets to find surface area), $7^{\text {th }}$ grade (solve problems involving scaled drawings of geometric figures and describe $2 \mathrm{D}$ figures that result from slicing $3 \mathrm{D}$ figures), and $8^{\text {th }}$ grade (understand that a 2D figure can be obtained from a sequence of rotations, reflections, translations, and dilations). 


\title{
WORKSHOP PROPOSAL FORM
}

\author{
2015 Annual ASEE K-12 Workshop on Engineering Education \\ "Authentic Engineering: Representing \& Emphasizing the E in STEM" \\ Presented by Dassault Systems \\ Saturday, June 13, 2015 \\ 8:00 A.M. - 5:00 P.M. \\ Sheraton Seattle $\mid$ Seattle $\mid$ WA
}

Diversity. This year is the American Society for Engineering Education's "Year of Action on Diversity." It is essential that we have a diverse engineering workforce to solve diverse problems. To do that and to have an engineering-literate public, it is essential that we reach every preK-12 student with high-quality engineering education, drawing on issues of access and equity in the classroom and in the curriculum. Reviewers would like to know how your proposed workshop will address diversity.

Provide a description of how you will explicitly address diversity - e.g., diversity with respect to gender/sex, ethnicity or race, special education inclusion, socio-economic status, or LGBT status - in your workshop (maximum 2,000 characters):

Spatial Visualization (SV) skills have been correlated with improved performance in STEM, but women and other underrepresented minorities (URMs) in STEM have on average lower SV skills. In fact, SV training was highlighted as an approach to increase diversity in STEM, in a 2010 report "Why So Few? Women in Science, Technology, Engineering, and Mathematics"1. To address this disparity Sorby, et al. ${ }^{2}$ developed a one-credit college level course in SV, and it has proven to effectively raise GPAs in the target demographics for a wide range of STEM courses.

SV skills are believed to develop at young ages through games such as block building and playing with Lego ${ }^{\mathrm{TM}}$. In addition, drawing is already emphasized in grades K-8. The SpatialKids ${ }^{\mathrm{TM}}$ App will allow students from all backgrounds to develop SV skills at an age where it can be easily acquired reducing any obstacles that women and other URMs often face at a later age. The ability to draw one's dream invention will attract more students to the STEM fields and reduce barriers for those who have been hesitant to become engaged.

It is recognized that many underserved communities do not have access to iPad technology. Therefore, to ensure that the application can be broadly disseminated, a web-based solution is currently being developed and will be provided to workshop participants when available. In addition, not all teachers are familiar with teaching SV. The integrated help and automatic grading in SpatialKids ${ }^{\mathrm{TM}}$ allow the software to be easily incorporated into the curriculum by teachers from a wide range of backgrounds.

1. "Why so Few? Women in Science, Technology, Engineering and Mathematics". Published by AAUW, ISBN: 978-1-879922-40-2, 2010.

2. Sorby, S. A., \& Veurink, N. (2010). Long-term Results from Spatial Skills Intervention among First-Year Engineering Students. In Proceedings of the 65th Midyear Meeting of the Engineering Design Graphics Division of ASEE. 


\section{WORKSHOP PROPOSAL FORM}

2015 Annual ASEE K-12 Workshop on Engineering Education

"Authentic Engineering: Representing \& Emphasizing the E in STEM"

Presented by Dassault Systems

Saturday, June 13, 2015

8:00 A.M. - 5:00 P.M.

Sheraton Seattle $\mid$ Seattle $\mid$ WA

Are there any online components to the proposal or presentation? (Note that these online components may only be available to presenters or those who have their wireless subscriptions, since wireless may not be available during the workshop sessions.)

$\triangle$ No

$\square$ Yes

Please describe: Our set of training iPads will be preloaded with the SpatialKids ${ }^{\mathrm{TM}}$ software, so an Internet connection is not required for the workshop. If an Internet connection is available we can have participants download the complementary software onto their own devices during the workshop; otherwise they will do this on their own at a later date. In an actual K-12 setting, teachers will use the Internet to setup student accounts and view each student's progress. If an Internet connection is available in the workshop, then we will demonstrate these features live, otherwise we will demonstrate these features with pre-saved data. In an actual K-12 classroom, Internet is not actually needed when students are working on the App. The grading algorithm for each activity is stored locally on each iPad. The data produced from the sketches (number of attempts, number of hints, number of peeks at the solutions...) are synchronized automatically when the teacher brings the iPads to a WiFi area.

Grade Level Target Audience (check all that apply):

$\triangle$ Primary (EC-2)

$\triangle$ Elementary (3-5)

$\triangle$ Middle School (6-8)

High School (9-12)

Maximum Number of Participants: 20

We will bring 20 iPads. If participants have their own iPads, we can increase the number of participants and install the software on their individual devices.

If this number is greater than 25 , please describe how your workshop will equally engage all participants.

By nature, the iPad App is extremely hands-on and engaging. Each participant will have access to his or her own device to play with the software.

All Seating is Classroom (tables and chairs).

Audio Visual Equipment Requests: 


\section{WORKSHOP PROPOSAL FORM}

2015 Annual ASEE K-12 Workshop on Engineering Education

"Authentic Engineering: Representing \& Emphasizing the E in STEM"

Presented by Dassault Systems

Saturday, June 13, 2015

8:00 A.M. - 5:00 P.M.

Sheraton Seattle $\mid$ Seattle $\mid$ WA

Note: An LCD projector, screen and podium with attached microphone are provided. Requests for additional equipment or resources (e.g., internet connection or laptops) will incur extra charges. If you do not have additional requests, please indicate with "Not applicable."

Depending on the cost, we may request internet connection if many participants sign up and want to bring their own iPads. We will need internet to install the software on their devices.

\section{Reminder:}

Presenters must register and pay the registration fee to support their workshop attendance and audio/video costs.

Thank you for completing this proposal form!

Please review this document prior to submitting it to ensure that all items are complete.

Date Received:

Received By:

Proposal ID Number: 